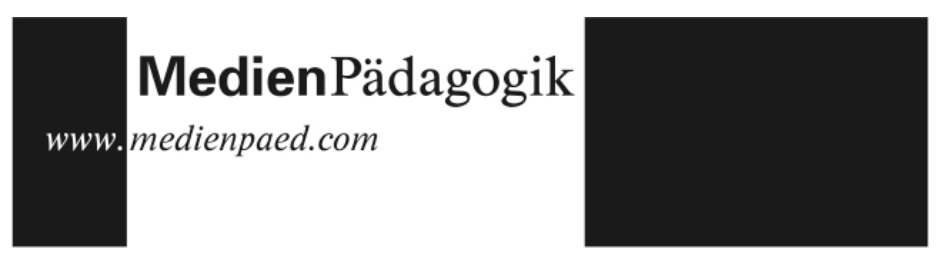

Rezensionen

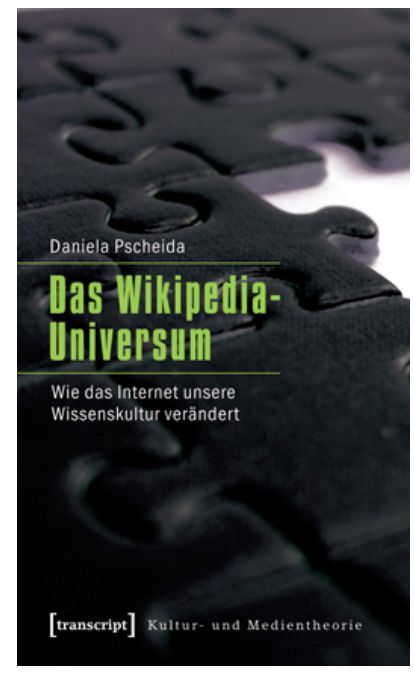

Daniela Pscheida

Das Wikipedia-Universum

Wie das Internet unsere Wissenskultur verändert

Bielefeld: transcript Verlag, 2010. 522 Seiten

ISBN: 978-3-8376-1561-6

$€ 29.80$; CHF 36.80

\title{
Wikipedia - Spiegel einer neuen Wissenskultur?
}

"Wikipedia ist ein Projekt zum Aufbau einer Enzyklopädie aus freien Inhalten in allen Sprachen der Welt. Jeder kann mit seinem Wissen beitragen.» ${ }^{1}$

Wikipedia ist zweifelsohne eine der prominentesten Anwendungen des Web 2.0. In ihr scheinen sich die Visionen Brechts und Enzensbergers von einer direkten demokratischen Partizipation am Prozess medialer Produktion zu realisieren. Doch führen diese und andere neue Entwicklungen des Internets tatsächlich zu einem gesamtgesellschaftlich veränderten Umgang mit Wissen und Wissensproduktion? Sind wir womöglich auf dem Weg zu einer «Wissenskultur der Amateure und Laien» (S. 11), wie es sich in der feuilletonistischen Debatte spiegelt?

Mit ihrem 〈Wikipedia-Universum〉 rekurriert die Medien- und Kommunikationswissenschaftlerin Daniela Pscheida auf McLuhans "Gutenberg-Galaxis» (1962) und damit verbunden auf die Tatsache, dass sich im Kontext des Buchdrucks und der gesellschaftlichen Entwicklungen der Frühen Neuzeit schon einmal ein Wandel der Wissenskultur vollzogen hat. Vor dem Hintergrund ihrer These «von einer Parallelität bzw. gleichartig gelagerten Potentialität zwischen aktuellen soziokulturellen und medialen Konstellationen und jenen sich zwischen Renaissance und Aufklärung im frühneuzeitlichen Europa ereignenden Prozessen» (193) nähert sie sich schließlich der Frage nach einer erneuten Veränderung der Wissenskultur im 21. Jahrhundert systematisch an.

\footnotetext{
${ }^{1}$ http://de.wikipedia.org/wiki/Wikipedia:Hauptseite [Zugriff: 13.11.2011]
} 
Die Grundlage ihrer Argumentation bildet die Auseinandersetzung mit dem Wissensbegriff sowie dem Verhältnis von Wissen, Medien und Wissenskultur im historischen Wandel (Kapitel I). Vertieft wird dieser theoretische Exkurs durch eine analytische Kontext-Betrachtung soziokultureller Bestrebungen (Individualisierung, Säkularisierung und Rationalisierung) und medialer Veränderungen (Verbreitung des Buchdrucks) der Frühen Neuzeit auf der Basis eines von ihr entwickelten mehrdimensionalen Analysemodells (Kapitel II). Dieses ist betont koevolutionär angelegt, denn ein wissenskultureller Wandel bilde sich schließlich «erst aus dem Wechselspiel zwischen Medium und Gesellschaft»(86) heraus. Ihre Kontextanalyse schließt Pscheida mit der Darstellung der sukzessiven Institutionalisierung wissenschaftlicher Forschung und neuer Formen des Wissenstransfers zwischen Wissenschaft und deren Öffentlichkeit (Wissenschaftspopularisierung) sowie mit der Explikation des Wahrheitsmodells als Wissensmodell der typografischen Ära. Enzyklopädien als zentrale Medienprodukte dienen Pscheida dabei als Anhaltspunkt, da sich in innen die wissenskulturellen Spezifika manifestieren würden.

Im zweiten Teil der analytischen Annäherung (Kapitel III) zeichnet Pscheida mit Bezug auf die gesellschaftlichen Makro-Trends Globalisierung, Individualisierung und Demokratisierung zunächst die zeitgeschichtlichen Veränderungen im Übergang von der Moderne zur Postmoderne nach, die verbunden mit der zunehmenden Wissensorientierung westlicher Gesellschaften und technologischen Weiterentwicklungen per se hinreichende Voraussetzungen für einen erneuten Wandel der Wissenskultur darstellen (könnten). Auch die Diskussion um die Begriffe 〈Wissensgesellschaft) und (Modus 2〉 (Gibbons et al. 1994) spiegelt eine hierfür geeignete Konstellation. Beide Konzepte verweisen sowohl auf eine Omnipräsenz und gewisse Abhängigkeit von Medien und (wissenschaftlichem) Wissen als auch auf ein verändertes Verhältnis von Wissenschaft und Öffentlichkeit, insofern (1.) die Öffentlichkeit potentiell (!) eine zunehmend aktivere Rolle in der tradierten sozialen Praxis der Wissensproduktion und -popularisierung einzunehmen und (2.) sich der einstige Wissenschaftsethos (insbesondere der Anspruch auf 
Wahrheit, Objektivität, Rationalität und Expertentum) im Prozess selbstgesteuerten Wissensmanagements zu verändern vermag.

Im vierten Kapitel widmet sich Pscheida darauf aufbauend explizit den medialen Veränderungen am Ende des 20. und Beginn des 21. Jahrhunderts: Das Internet sei als Leitmedium der digitalen Wissensgesellschaft zu begreifen, dessen revolutionäres Potential in den vorhandenen Beteiligungsstrukturen (insbesondere des Web 2.0) liegt, die wiederum Möglichkeitsräume für einen demokratischen den gesellschaftlichen Wissensdiskurs offerieren. Am Beispiel Wikipedia zeigt die Autorin anschließend mit den Ergebnissen ihrer rekonstruktiven Betrachtung, dass zwar tatsächlich «eine Liberalisierung und Ausweitung der Inhalte einerseits und eine Demokratisierung der Beteiligungsmuster andererseits» (393) deutlich werden, dass eine vertiefende Analyse jedoch gleichsam auf die Existenz hierarchischer Machtverhältnisse verweist, die basisdemokratischen Handlungen und damit einer wissenskulturellen Wende entgegenstehen (Kapitel V). Zumindest ein Wandel, der «auf die Etablierung grundsätzlich anders gelagerter Prinzipien und Konventionen in Bezug auf Wissen und damit schließlich auch auf tiefenstrukturelle Veränderungen des gesamten Wissensmodells» (413f.) verweist, sei jedoch zu konstatieren (Kapitel $\mathrm{VI})$.

Insgesamt sei das Ergebnis der (medien-)historischen Rekonstruktion und exemplarischen Wikipedia-Analyse damit Anlass genug, die Wissenskultur am Beginn des 21. Jahrhunderts nicht mehr länger ausschließlich vor der Folie des klassischen Wissensmodells zu diskutieren, sondern zu realisieren, dass im wissenschaftlichen Diskurs, aber auch in der Praxis der Bildungsinstitutionen Schule und Universität weitere Modelle zu berücksichtigen sind (Kapitel VII).

Pscheidas 'Wikipedia-Universum) reiht sich nicht einfach ein in die bereits zahlreich erschienenen Wikipedia-Studien; vielmehr handelt es sich hierbei um eine systematische Auseinandersetzung mit der Bedeutung des Internets für unsere Wissenskultur. Mit ihren Ausführungen greift die Autorin so auch eine Debatte auf, die weit über die Diskussionen um Potentiale und 
Herausforderungen des universellen Mediums Internet hinausgehen. Stattdessen werden strukturelle Veränderungen in den Blick genommen, die unsere Gesellschaft langfristig prägen werden und sich daher schon jetzt in der Reflexion unserer Denk- und Deutungsmuster sowie in unserem (pädagogischen) Handeln niederschlagen sollten.

Über diese Anregungen hinaus gelingt es der Autorin, einen fundierten Überblick über den interdisziplinären Forschungsstand zum Thema und durch zahlreiche Exkurse gleichsam Anstöße für weiterführende Recherchen zu geben. Ihrer komplexen Argumentation kann dank vieler Zusammenfassungen während der gesamten Lektüre problemlos gefolgt werden, was gleichsam die Möglichkeit einräumt, sich je nach Interesse ausgewählten Kapiteln isoliert zuzuwenden.

Claudia Kuttner

\section{Literatur}

Brecht, Berthold. Der Rundfunk als Kommunikationsapparat. Rede über die Funktion des Rundfunks. In: Claus Pias, Joseph Vogel, Lorenz Engell, Oliver Fahle, Britta Neitzel (Hrsg.): Kursbuch Medienkultur. Die maßgeblichen Theorien von Brecht bis Baudrillard. 5. Aufl. Stuttgart: DVA, 2004 [1932], 259-263.

Enzensberger, Hans Magnus. Baukasten zu einer Theorie der Medien. In: Peter Glotz, Hrsg. Baukasten zu einer Theorie der Medien: Kritische Diskurse zur Pressefreiheit. München: Fischer, S. 97-132.

Gibbons, Michael; Limoges, Camille; Nowotny, Helga; Schwartzmann, Simon; Scott, Peter; Trow, Martin. The New Production of Knowledge. The Dynamics of Science and Research in Contemporary Societies. London u.a.: Sage, 1994.

McLuhan, Marshall. Die magischen Kanäle: Understanding Media. Düsseldorf u.a.: Econ, 1992 [1964]. 